\title{
Single layer printed reflectarrays at MM-Waves
}

\author{
J. Lanteri*, J. Y. Dauvignac, Ch. Pichot, C. Migliaccio* \\ Laboratory of Electronics, Antennas and Telecommunications (LEAT), University of Nice-Sophia Antipolis, CNRS, Sophia-Antipolis, \\ France
}

\section{Email address:}

Jerome.lanteri@unice.fr (J. Lanteri), claire.migliaccio@unice.fr (C. Migliaccio)

\section{To cite this article:}

J. Lanteri, J. Y. Dauvignac, Ch. Pichot, C. Migliaccio. Single Layer Printed Reflectarrays at MM-Waves, Journal Electrical and Electronic Engineering. Vol. 1, No. 1, 2013, pp. 20-28. doi: 10.11648/j.jeee.20130101.12

\begin{abstract}
This paper talks about a method of conception and design constraints on mm-wave reflectarrays. The developed tool allows us to plan quickly the behavior of large reflectarray (several tens of wavelength) according to parameters as illumination law or manufacturing tolerance with good agreement with measurements. An ultra-low side-lobe reflectarrays of $130 \mathrm{~mm}$ diameter is designed. The structure combines the advantages of a reflectarray with an offset source and those of a specific primary source, exhibiting a prolate radiation pattern, having very low side lobe levels. The maximum gain obtained at $94 \mathrm{GHz}$ is $40 \mathrm{dBi}$ and the side-lobe level is inferior to $-28 \mathrm{~dB}$. Finally, a simultaneous multi-lobe antenna is designed at 94 GHz. The primary source is an open-ended waveguide and the phase profile is calculated by the program introduced in the first part. In this case, the four main lobes are placed in the same plane and for equal to $-30,-10,10,30^{\circ}$. This reflectarray can be used for actual and future generations of automotive radar. The first obtained results are encouraging and show the validity of the concept. Solution retained here is a low-cost solution. The proposed structures are developed on a single layer substrate and fabricated using standard photolithographic techniques. The aim of this article is to show that we can obtain interesting results with relatively simple and low-cost solutions, but also to show the limits of these type of solutions.
\end{abstract}

Keywords: Reflectarray, Reflector Antenna, Reflector Antenna Feed, Mm-Wave Arrays Antenna

\section{Introduction}

Millimeter-wave reflectarrays have been of increasing interest over the past two decades [1]. Research and developments have been pushed by the demand for high frequency systems due to the low-frequency spectrum congestion. In addition, the use of mm-Waves enables the design of compact platforms with high resolutions which make sense for radar applications. The $77 \mathrm{GHz}$ automotive radar [2] with a moderate $200-300 \mathrm{~m}$ detection range is one of the examples. Recently, the bandwidth has been extended to 76-81GHz. For long-range detection, $94 \mathrm{GHz}$ is better due to the lower atmospheric attenuation [4]. For the antenna designer, the requirements are similar: high gain (min. 30 $\mathrm{dBi}$ ), low side lobes, low profile, low weight, and not to be neglected, low cost. The reflectarrays have the better match to these constraints because they combine the advantages of quasi-optic and printed antennas. Classical printed circuits techniques are used for low cost purposes [5-6].

Nevertheless, the patches etched on the substrate are small in size and often reach technological limits due to the small wavelength value.

In this paper, we want to discuss the possibilities, limita- tions and improvements for single layer printed reflectarrays in W-band in terms of design rules and performances. We deliberately choose to use rectangular patches because of their simplicity although sophisticated cells [7-8-9] can provide complete phase range covering, including at $\mathrm{mm}$-Waves. All the antennas are designed to work at $94 \mathrm{GHz}$, because the field of applications of our investigations was initially the collision avoidance radar for helicopters.

The paper first describes an in-house modeling program based on an equivalent aperture method. Validations and limitations are presented.

Secondly, the impact of manufacturing tolerances is discussed together with performances of reduced cell size. Examples are taken with cells in $\lambda / 2$ and $\lambda / 4$.

In a third part, we present a low-cost solution for obtaining an ultra low-side lobes, high efficiency and high bandwidth reflectarray antenna. Finally, a multi-lobe reflectarray is presented. This kind of antenna can be useful for radar applications in particular in automotive domain.

\section{Reflectarray Modeling}

Reflectarray are electrically large antennas with small 
elements. Therefore, reflectarray modeling is a multi-scale problem that requires high simulations resources [10]. These limit the reflectarray size for full-wave simulations. In the present paper, we aim to design reflectarrays for long range mm-Wave radar applications, i.e. typically 35-40 $\mathrm{dBi}$ gain. The corresponding reflectarray size reaches up to $50 \lambda$ that is by far too large for a full wave simulation. Alternative simulations including approximations can be done in order to overcome this difficulty. Therefore, we have developed a reflectarray modeling program based on the equivalent aperture method (EAM).

\subsection{Radiation Pattern Calculation}

Before going into the EAM developments, it is important to set up what can be taken into account and what is neglected in the modeling. Information is sum-up in Table 1.

Table 1. Reflectarray Modeling Assumptions

\begin{tabular}{ll}
\hline Taken into account & Neglected \\
Amplitude of the PF* & Coupling between cells \\
Phase of the primary feed & Edge diffraction \\
Aperture blockage & Surface waves \\
Spillover & Ohmic and dielectric losses \\
Primary feed offset (if some) & PF off-aperture radiation \\
\hline
\end{tabular}

* Primary Feed

The reflectarray surface is divided into elementary cells as shown in Fig. 1.a. These cells are square and indexed by the integers $(i, j)$ : cell $(i, j)$. They are composed of a metallic patches etched on a dielectric substrate with a ground plane (Fig. 1.a and 1.b). The cell size (d) depends on the choice made by the reflectarray designer. Most antennas presented in this paper are working with $d=\lambda / 2$ although an example with smaller cells is discussed in section 3 .

The radiated far field of the reflectarray is the superposition of the radiated far field by each cell $(i, j)$ :

$$
\vec{E}_{r a y}=\sum_{i, j=1,1}^{N, N} \vec{E}_{r a y_{i j}}
$$

The EAM consists in modeling the elementary cell radiated far field by the one of its equivalent aperture, i.e. the aperture with the same surface:

$$
\vec{E}_{r_{i j}}=2 j \pi \frac{e^{-j k r}}{k r} \vec{u} \times\left(\widetilde{\vec{E}}_{0 t_{i j}}(\alpha, \beta) \times \vec{u}_{z}\right)
$$

where

- $(r, \theta, \varphi)$ are the spherical coordinates as defined on Fig. 1.a

- $\vec{u} \rightarrow$ is the unit vector of the spherical coordinates defined by: $O M=r \vec{u}$

- $\widetilde{\vec{E}}_{0 t_{i j}}(\alpha, \beta)$ is the 2D Fourier transform of the tangentialelectric field located in $z=0^{+}$.

$$
\widetilde{\vec{E}}_{0 t_{i j}}(\alpha, \beta)=\frac{1}{\lambda^{2}} \iint \vec{E}_{0 t_{i j}}(x, y) e^{j k(\alpha x+\beta y)} d x d y
$$

with:

$$
\begin{aligned}
& \text { - }\left\{\begin{array}{l}
\alpha=\sin \theta \cos \varphi \\
\beta=\sin \theta \sin \varphi
\end{array}\right. \text { are the spectral coordinates } \\
& \text { - } \vec{E}_{0 t_{i j}}(x, y)=0 \quad \text { outside the cell }(i, j) \\
& \text { - } \vec{E}_{0 t_{i j}}(x, y)=A_{i j} e^{j \varphi} \vec{u}_{y} \text { inside the cell }(i, j)
\end{aligned}
$$

where is $A_{i j}$ the real coefficient that accounts for the primary feed amplitude distribution on the elementary cell. The phase $\varphi_{i j}$ is the total phase of the cell that is the algebric sum of:

- The primary feed phase distribution,

- The propagation delay due to the path of the incident wave $\left(\varphi_{\text {inc }}\right)$ from the phase center of the primary feed to the cell $(i, j)$,

- The propagation delay due to the path of the reflected wave $\left(\varphi_{\text {ref }}\right)$ from cell $(i, j)$ to the outgoing plane wave,

- The phase of the cell itself $\left(\varphi_{\text {cell } i j}\right)$ that has been adjusted with the patch dimensions for obtaining the foreseen radiation pattern.

In addition, the electric field of the primary feed is linearly polarized. We assume that it is along Oy. This simplified model corresponds to an open-ended waveguide as well as to small horns that are typically used as primary feeds in the examples presented in this paper. As the electric field $\vec{E}_{0 t_{i j}}(x, y)$, i.e Aij and ij are constant, is supposed to be constant in a cell, its 2D-Fourier transform will be described by a sinc function.

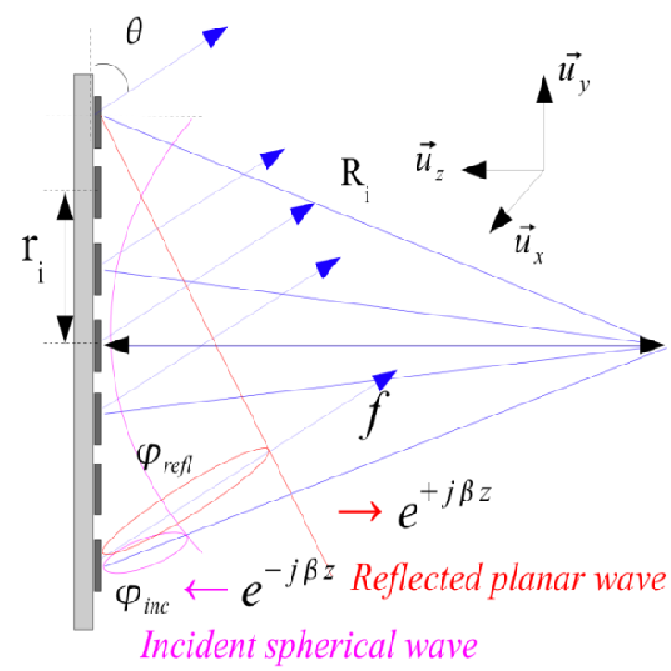

Fig. 1.a. Side view 


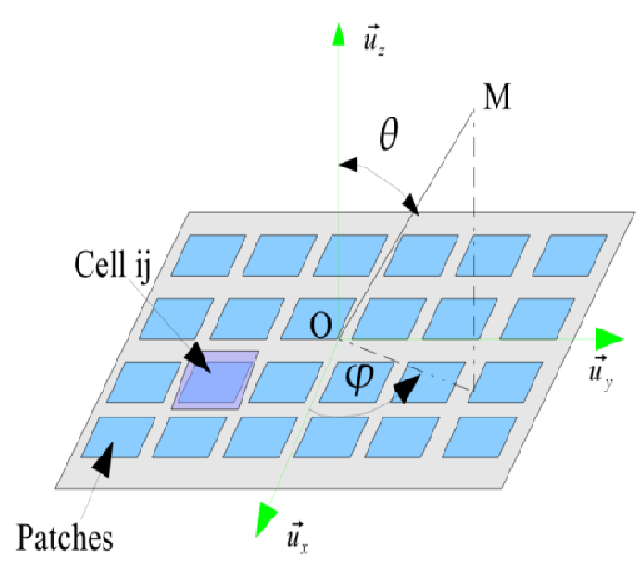

Fig. 1.b. Front view

Fig. 1. Reflectarray decomposition in to cells

\subsection{Programming Considerations and Parseval's Theo- rem} [11].

The program is implemented with the free software Scilab

As we aim to design very large reflectarrays, it is important to ensure a fast calculation. For this purpose, we have chosen to develop an analytic expression for the 2D Fourier transform of each cell. In addition, according to the simplicity of the modeling, especially considering all the important neglected effects, the objective is to be accurate for the main lobe and the first side lobes levels. Therefore we have chosen not to use the implemented 2D Fourier transform models built in scilab but to use an analytic formula with a dense sampling in $\theta$ around the main lobe and smaller one outside. The simulation time can be greatly reduced at the expanse of the out-of-main lobe radiation accuracy.

This can be easily explained considering equations (4.1 and 4.2) of the tangential electric field on the cell. It is alike a rectangular function whom $2 \mathrm{D}$ Fourier transform is a product of cardinal sinus (sinc function). The highest the sampling interval, the better is sinc secondary lobes restitution.

In order to determine the best compromise between the number of sampling points and the program accuracy, we have decided to add a module for Parseval's theorem verification.

Applied to EAM, Parseval's theorem expresses that, under certain conditions, the radiated power, $P_{\text {ray }}$, is the same as incident power coming from the primary feed "intercepted" by the reflectarray surface $P_{i n c}$. This can be formulated by equations (5) to (7):

$$
P_{i n c}=\iint_{S} \frac{\vec{E}_{0 t}(x, y) \bullet \vec{E}_{0 t}^{*}(x, y)}{2 \eta_{0}} d x d y
$$

$$
P_{\text {ray }}=\iint_{\text {Sphere,radius } r} \frac{\vec{E}_{\text {ray }} \bullet \vec{E}_{\text {ray }}^{*}}{2 \eta_{0}} r^{2} \sin \theta d \theta d \varphi
$$

Parseval's theorem expression to the antennas:

$$
\iint_{S} \vec{E}_{0 t}(x, y) d x d y=\iint_{\text {Sphere }} \frac{\vec{E}_{\text {ray }} \bullet \vec{E}_{\text {ray }}^{*}}{2 \eta_{0}} r^{2} \sin \theta d \theta d \varphi(7)
$$

The related conditions for its applications are:

- That the antenna radiates perpendicular to the reflectarray surface (case $\theta=0$ ),

- That the antenna is large enough: same as saying that it is directive.

The second condition is always satisfied since we aim to work up to $50 \lambda$ diameter refelctarrays. The first one means that the validity of Parseval's theorem has to be checked with centered configurations with pencil beam in $\theta=0$. Therefore we have chosen to check the Parseval's theorem with a $130 \mathrm{~mm}$ diameter reflectarray with focal to length ration of 0.5 operating at $94 \mathrm{GHz}$. Table II sums up the results for different sampling intervals, the error percentage is calculated by:

$$
E=100 *\left|\frac{P_{i n c}-P_{r a y}}{P_{i n c}}\right|
$$

$P_{i n c}$ is calculated by simply adding the coefficient $A_{i j}$ and $P_{\text {ray }}$ is calculated from the integration of the radiated power density. For simplifying the integration and the computation time, the integration is approximated by the method of rectangles. In this case, the accuracy depends on the number of sampling points taken in $\theta$. As the main lobe mostly contributes to the radiation, the sampling interval is chosen to be different inside the main lobe and outside. Table II sums up the results for a $41 \lambda$ diameter reflectarray at 94 $\mathrm{GHz}$.

Table 2. Parseval's Theorem verification

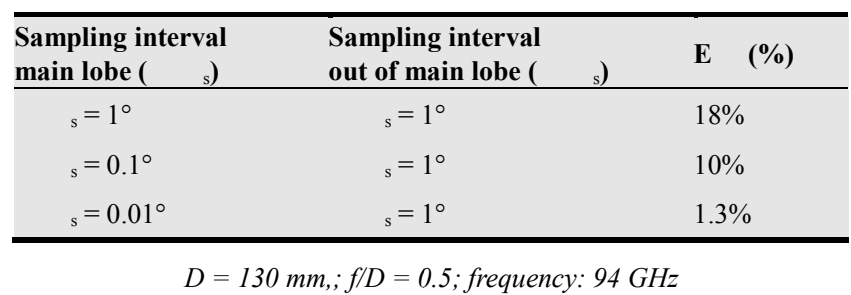

Parseval's theorem is verified with a satisfying accuracy of $1.3 \%$ for a dense sampling interval of the main lobe. The main lobe is defined over $\theta=0 \pm 5^{\circ}$. Interesting is that we can keep a relatively large interval value of $\Delta \theta_{\mathrm{s}}=1^{\circ}$ outside the main lobe. Overall simulation time does not exceed 3 minutes for a 5000 cells reflector on a standard notebook.

\subsection{Overall Design Procedure}

The design procedure of the reflectarray is the result of the 
association of the EAM for computing the radiation pattern and the determination of the cells phase distribution: $\left\{\varphi_{\text {cell }} i j\right\}$. In the case of a centered primary feed with a constant phase distribution on the reflectarray aperture and a desired radiation pattern having a pencil beam, the overall phase distribution $\left\{\varphi_{i j}\right\}$ is uniform and the phase distribution of the cells $\left\{\varphi_{\text {cell } \_j j}\right\}$ can be calculated from the phase equation compensation given in [1]. In other cases, like sectorial or squared cosec patterns the phase distribution has to be calculated by more sophisticated methods like the phase only synthesis [12].

Once the phase distribution has been setup, patches dimensions have to be adjusted accordingly. They are obtained from a set of simulations using ANSOFT-HFSS and the periodic structure module with magnetic and electric walls (perfect E - perfect $\mathrm{H}$ ) as described in [13].

Fig.2 is the flowchart of the complete design procedure including the radiation pattern calculation. It corresponds to the "pencil beam case". In case of a non-standard radiation pattern, the design procedure is greatly changed but the radiation pattern still can be calculated by entering $\left\{\varphi_{\text {cell } \_i j}\right\}$ externally.

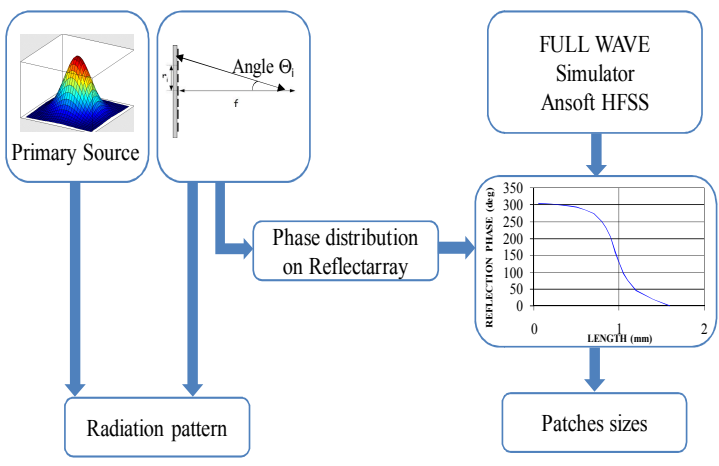

Fig. 2. Flowchart for the radiation pattern computation

\subsection{Validation and Performances}

For validation purpose, a $130 \mathrm{~mm}$ diameter offset reflectarray has been designed and measured at $94 \mathrm{GHz}$. The focal length over diameter ratio of 0.5 at $94 \mathrm{GHz}$ using a single layer structure with rectangular patches etched on Duroid dielectric substrate $\left(\varepsilon_{\mathrm{r}}=2.2, \mathrm{~h}=381 \mu \mathrm{m}\right)$. The primary feed is an open-ended WR-10 waveguide. According to the simplicity of the modeling and considering all the important neglected effects, the objective is to be accurate for the main lobe and the first side lobes levels. Comparisons between simulations and measurements are shown in Fig. 3. More details on the reflector design and its performances will be given in section 3 .

A good concordance between simulations and measurements is observed with respect to the main lobe and the first side lobes level $\left(\theta= \pm 15^{\circ}\right)$. Furthermore, we can observe that the accuracy is very good for $\theta= \pm 5^{\circ}$ and that a shift in the lobes positions appears above. This result was expected since the sampling interval in $\theta$ increases outside of the main lobe (i.e $\theta= \pm 5^{\circ}$ ) and does not match the variations of the sinc function anymore. Same conclusions are true for the
E-plane except for a higher level measured around the $\theta=$ $30^{\circ}$. This is due to the masking effect of the waveguide used for connecting the primary feed to the measurement system.

The program has also been checked successfully on cosec-squared antenna. Results can be found in [14].

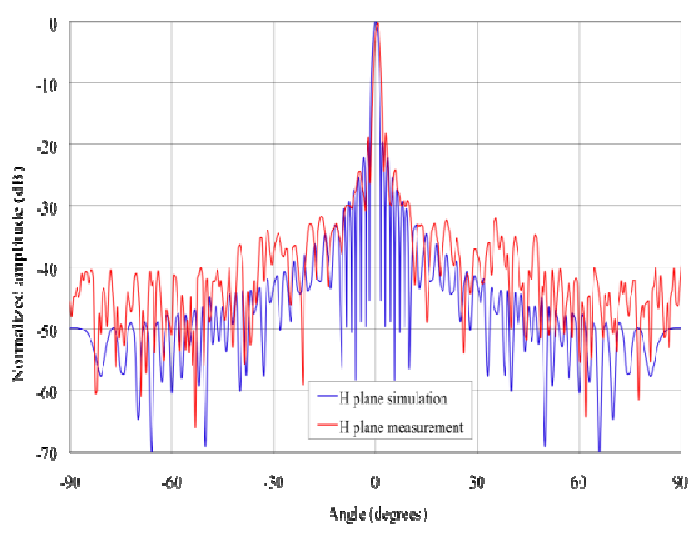

Fig. 3. a overall H-plane radiation pattern

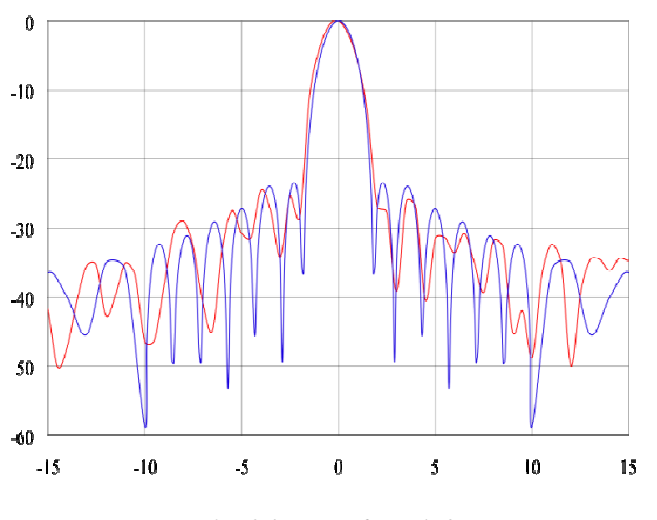

Fig. 3.b zoom $\theta= \pm 15^{\circ}$

Fig. 3. H-plane at $94 \mathrm{GHz}$. Reflectarray, $f / D=0.5, D=130 \mathrm{~mm}$.

\subsection{Fabrication Tolerances}

The tolerance of manufacturing is an important parameter in W-band. In a single layer design, all the possible patch dimensions are used as shown in Fig. 4. In particular those close to $100 \mu \mathrm{m}$ result in $50 \%$ error if we assume a fabrication tolerance of $\pm 50 \mu \mathrm{m}$ like in classical printed circuits fabrication techniques.

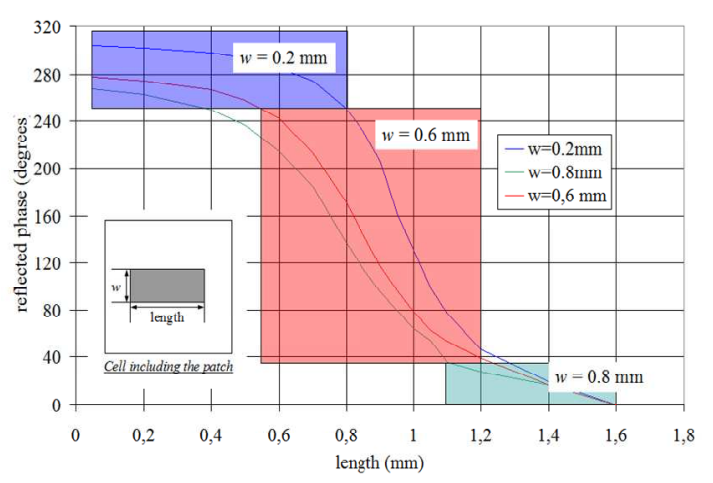

Fig. 4. Reflection phase versus patch width (w) and corresponding zones 
It is obvious that $\pm 50 \mu \mathrm{m}$ greatly changes the reflected phase of the cell in the resonant area. In order to quantify the phase discrepancy influence on the antenna radiation pattern, a pseudo random error sequence is applied on previously calculated patches dimensions by using the "prbs_a" function available in Scilab. The maximum error has to be defined by the user. In all following the examples, we chose $\pm 50 \mu \mathrm{m}$. Fig. 5 is the flowchart including the influence of the manufacturing tolerance.

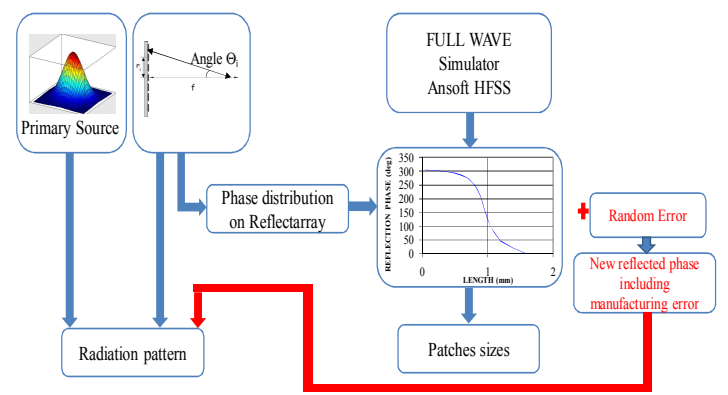

Fig. 5. Flowchart including manufacturing errors

In order to quantify the manufacturing tolerance impact a $15 \mathrm{~mm}$ and a $130 \mathrm{~mm}$ diameter reflectarrays are built and simulated with cell-size of $\lambda / 4 . \quad \lambda / 4$ cells are chosen because they are more sensitive to fabrication tolerance compared to $\lambda / 2$ cells.

Fig. 6 points out that manufacturing tolerances cause a loss in gain of $1 \mathrm{~dB}$ for the $130 \mathrm{~mm}$ diameter reflect array due to the unwanted changes of the phase distribution as shown in Fig. 7.

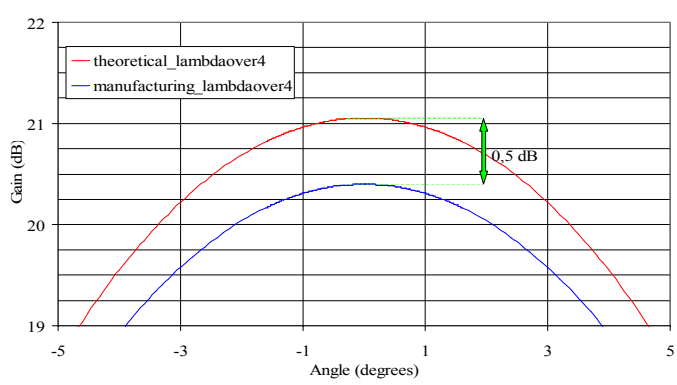

Fig. 6.a. Zoom in the main lobe for $D=15 \mathrm{~mm}$ at $94 \mathrm{GHz}$

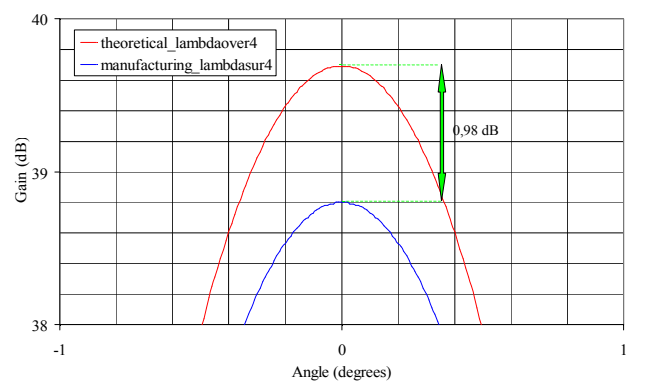

Fig. 6.b. Zoom in the main lobe for $D=130 \mathrm{~mm}$ at $94 \mathrm{GHz}$

Fig. 6. Fabrication tolerances versus reflectarray diameter and cell size Legends:- theoretical_lambdaover4 : without manufacturing tolerances; manufacturing_lamb-jaover4: with manufacturing tolerances

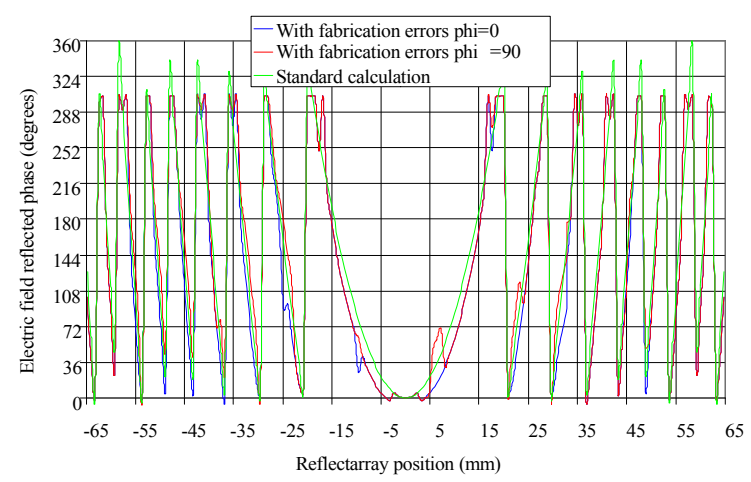

Fig. 7. Reflection phase with and without fabrication tolerances

Frequency: $94 \mathrm{GHz}, D=130 \mathrm{~mm}, f / D-0.5$

The mean square error MSE $\left(M S E=\sqrt{\frac{1}{N} \sum_{1}^{N}\left(\varphi_{\text {fabrication }}-\varphi_{\text {theoretical }}\right)^{2}}\right.$

with $\mathrm{N}$ the number of reflectarray's cells) is equal to $20^{\circ}$. As expected, fabrication tolerance is more critical for the 130 $\mathrm{mm}$ diameter reflectarray because the errors are repeated a larger number of time. For future reflectarray designs involving rectangular patches, it is necessary to minimize the influence of fabrication errors. A solution is to choose higher substrates that naturally provide smaller phase slopes. By this mean, a large fabrication error will result in a smaller phase discrepancy. Therefore we choose Duroid with $381 \mathrm{~m}$ height for future designs rather than $254 \mathrm{~m}$ or $127 \mathrm{~m}$ ones although the latest would have provided higher phase range covering.

\section{Ultra-low Side Lobes Reflectarrays}

Side lobe level is a critical issue for radar application. In addition, it is quite difficult to maintain them at low levels over a large frequency bandwidth. We propose a new approach based on use of primary sources having radiation patterns following the Prolate Spheroidal Function (PSF).

\subsection{Purpose of the Study}

The PFS was introduced by Slepian and Pollack in 1961 [15-16]. They form a complete orthogonal set. In signal processing, the low-order PSF's span the space of functions that are simultaneously concentrated in time and frequency. Applying a PSF window results in concentrating the lowest overall noise at the expense of the main lobe widening [17]. In other words, a diminution of the gain is expected while the "out of main lobe level" should drastically decrease. These functions are used in astronomy for coronography applied to the exo-planet detection. This structure is interesting for multi-beam application, when the system has several feeds. Considering the reflectarray structure, the illumination can be compared to a signal processing windowing. In this case finding a window that minimizes the secondary lobes is the same as designing a primary feed whose radiation pattern is PSF alike. Thanks to Orange software, SRSRD (designed for structures with symmetries of revolution and based on the formalism of Integral Equations in frequency domain) [18], a small stepped-horn was 
designed for this purpose. Its dimensions are shown in figure 10. They were chosen for fitting the PSF mask. More details can be found in [19].

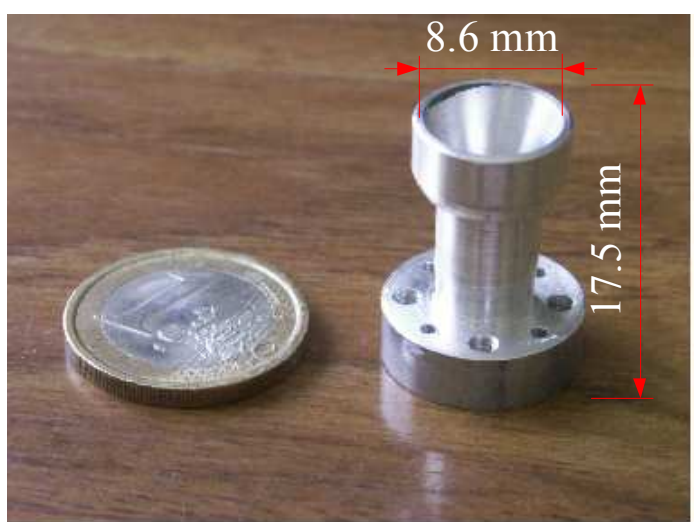

Fig. 10.a. Prolate horn antenna

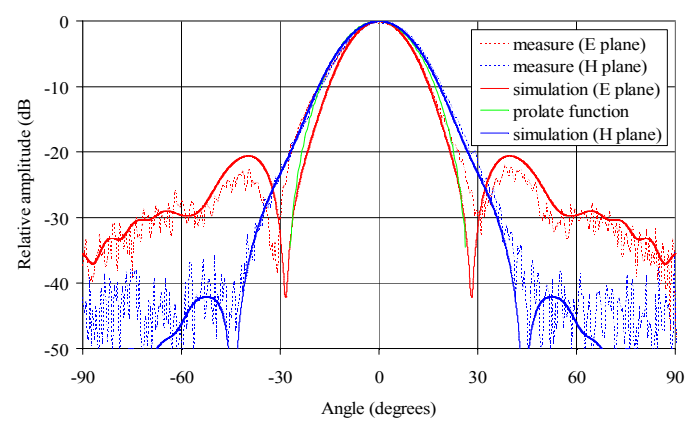

Fig. 10.b. Radiation pattern

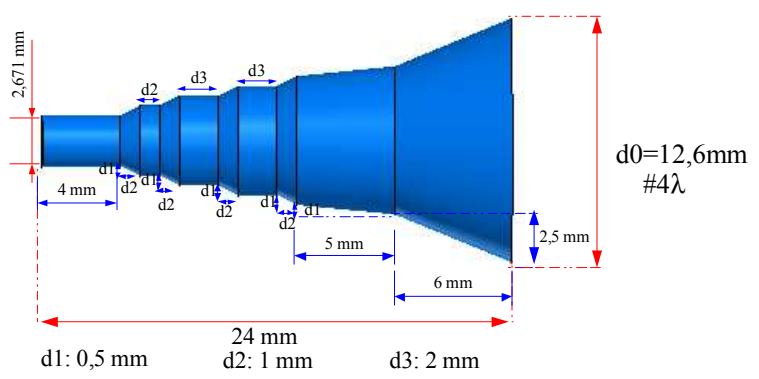

Fig. 10.c Prolate horn characteristics

Fig.10. Primary source

\subsection{Application and Restrictions}

Using a prolate horn as primary feed reduces the side-lobe level of the reflecarray. However, if the primary feed is centered, the aperture of the horn creates an important aperture blockage that neutralizes the expected improvements as shown in Fig. 11. This figure is the comparisons between the measured E-plane of a Fresnel reflector with an open-ended WR-10 primary feed and with a prolate one. Both of them are centered.

The E-plane was chosen because it has the highest secondary lobes. As expected from the PFS descriptions, the beamwidth is wider with the prolate horn. Nevertheless, due to the aperture blockage, the first side-lobes are not reduced accordingly. In order to take advantages from the PSF properties, we chose to design an offset fed reflectarray.

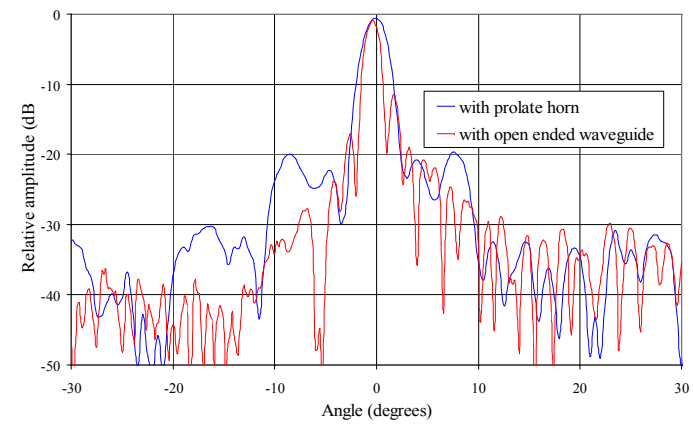

Fig. 11. Radiation pattern comparisons with a centered primary fee Printed Fresnel reflector antenna, $f / D=0.5, D=130 \mathrm{~mm}, 94 \mathrm{GHz}$

For this purpose, a $130 \mathrm{~mm}$ diameter antenna was designed with a primary feed placed at an offset of $-27^{\circ}$. The focal length over diameter ratio is equal to 1 . The antenna is shown in figure 12 and measurement results in figure 13.

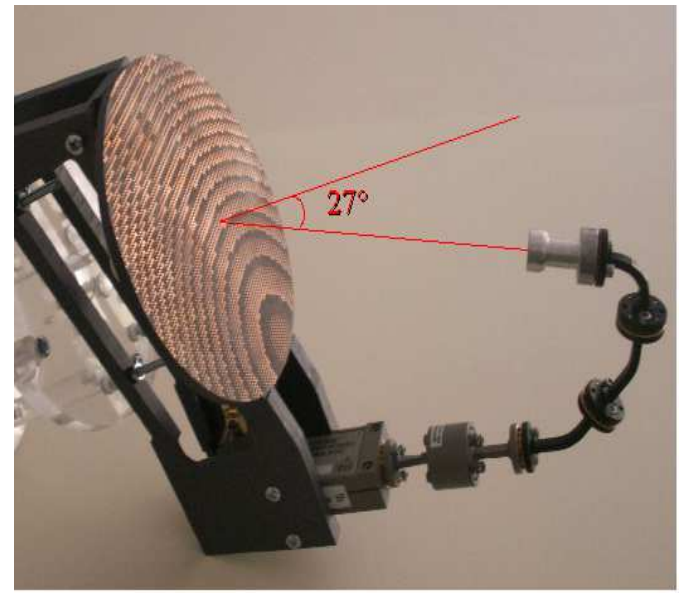

Fig. 12. Reflectarray with prolate horn primary feed placed at $-27^{\circ}$ offset Reflectarray, $f / D=0.5, D=130 \mathrm{~mm}$, main lobe at $0^{\circ}$

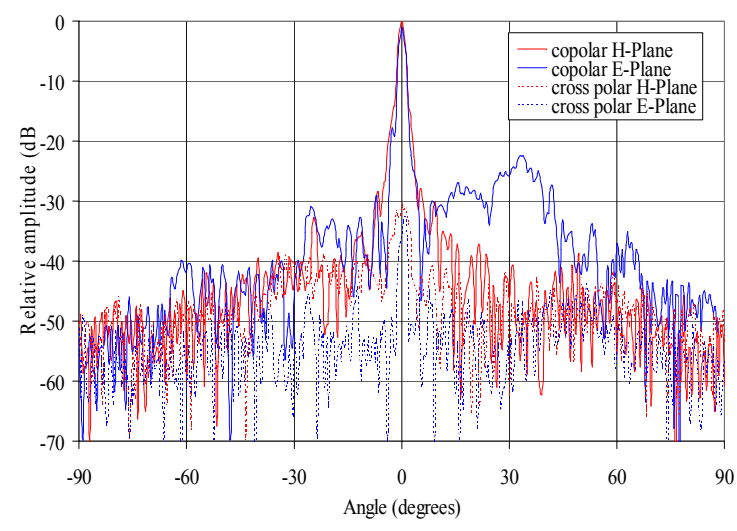

Fig. 13. Measured radiation pattern in the E-plane at $94 \mathrm{GHz}$

The influence of the prolate PF is obvious: first side lobe levels of E- and H-planes are about $-18 \mathrm{~dB}$ and $-28 \mathrm{~dB}$ respectively. Moreover, the other levels remain inferior to $-35 \mathrm{~dB}$. The offset solution has proved to be efficient for side 
lobe level reduction but the specular reflection creates a new peak of $-20 \mathrm{~dB}$ at $=30^{\circ}$. An improvement is proposed in section 3.4 .

\subsection{Gain Bandwidth}

Gain was measured between 80 and $100 \mathrm{GHz}$ in order to investigate the reflectarray bandwidth. One of the wellknown drawbacks of printed microstrip reflectarrays is their relatively narrow gain bandwidth, which is typically in the range of a few percents for a single layer design.

Radiation pattern measurements, that are not shown here, indicate that the first side-lobes level is stable between 92 and $96 \mathrm{GHz}$. In E-plane, an increase of the side-lobe level in the specular direction is observed. The gain evolution is represented in figure 16. The maximum gain $(39 \mathrm{dBi})$ is obtained at $94 \mathrm{GHz}$. The gain bandwidth at $-3 \mathrm{~dB}$ is $5 \%$ and maximum aperture efficiency, calculated for a circular aperture, $(\mathrm{a})$ is $48 \%$.

$$
\eta_{a}=\frac{G_{\text {measured }}}{(\pi D / \lambda)^{2}}
$$

\subsection{Improved Structure}

In order to avoid the specular reflection, we designed a second offset reflectarray with the main lobe pointing at $27^{\circ}$ (specular direction). Figure 14 is the radiation pattern measurements at $94 \mathrm{GHz}$ in the E-plane (which is the most critical for side lobe level). Due to the focusing in the specular direction, the specular side lobe radiation is "absorbed" by the main focus. Therefore, we expect an increase of the antenna gain.

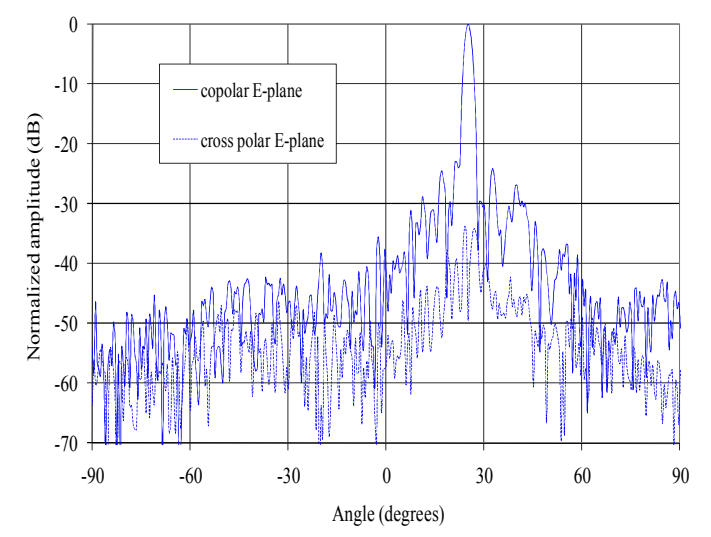

Fig. 14. Measured radiation pattern in the E-plane at $94 \mathrm{GHz}$ Reflectarray, $f / D=0.5, D=130 \mathrm{~mm}$, in specular direction $\left(27^{\circ}\right)$

Once again we have investigated the same evolution between 80 and $100 \mathrm{GHz}$. Results are presented on figure 15 . We can observe a good stability of the radiation pattern for these different frequencies in terms of gain or side-lobe levels.

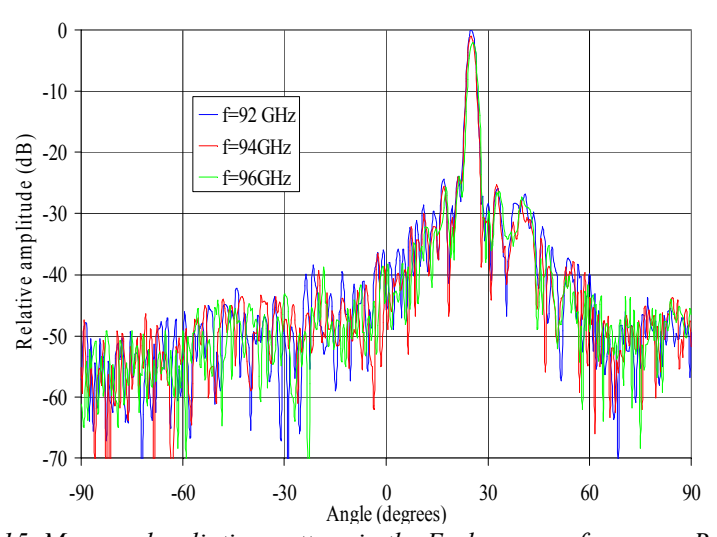

Fig. 15. Measured radiation pattern in the E-plane over frequency Reflectarray, $f / D=0.5, D=130 \mathrm{~mm}$, main lobe in specular direction $\left(27^{\circ}\right)$

The evolution of the gain for the $\mathrm{W}$-band is represented in figure 16.

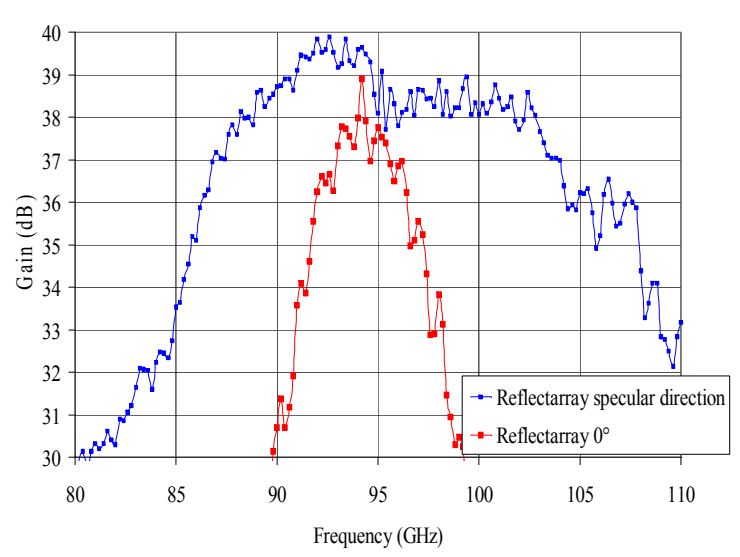

Fig. 16. Measured gain over frequency for both reflectarrays

As expected, the gain is increased of $1 \mathrm{~dB}$ (maximum gain: $40 \mathrm{dBi}$ ) that corresponds to an aperture efficiency of $61 \%$. The relative frequency bandwidth is about $17 \mathrm{GHz}(18 \%)$ which is three times larger than the one obtained with the previous reflectarray. These values are, to our best knowledge, state of the art at $94 \mathrm{GHz}$.

\section{Multi-lobes Reflectarrays}

The possibility to have multi-lobes or scanning antennas is important for the actual and future generations of automotive radars, and more generally, to increase the radiation pattern agility in order to match the more and more complex requirements assigned to the antennas. In order to demonstrate the reflectarray capabilities for this, we have designed a four simultaneous beam antenna working at $94 \mathrm{GHz}$ (figure 17). For this purpose, the primary feed is a centered open-ended waveguide and the phase profile is calculated with the reflectarray modeling program described in section 1. 


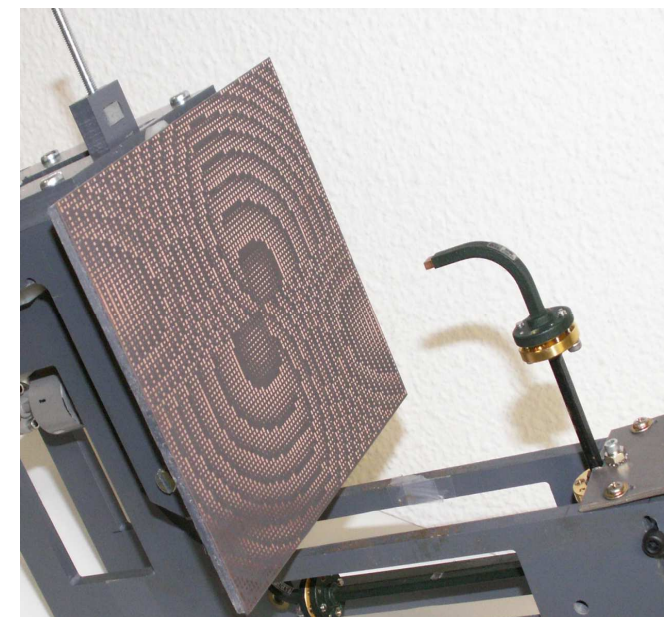

Fig. 17. Multi-lobe reflectarray 4 lobes in $\varphi=45^{\circ}$ plane with $\theta=-30^{\circ}, 0^{\circ}, 10^{\circ}$ and $30^{\circ}, D=130 \mathrm{~mm}, f / D=0.5$

The measured radiation pattern associated to the simulated radiation pattern is represented in figure 18 .

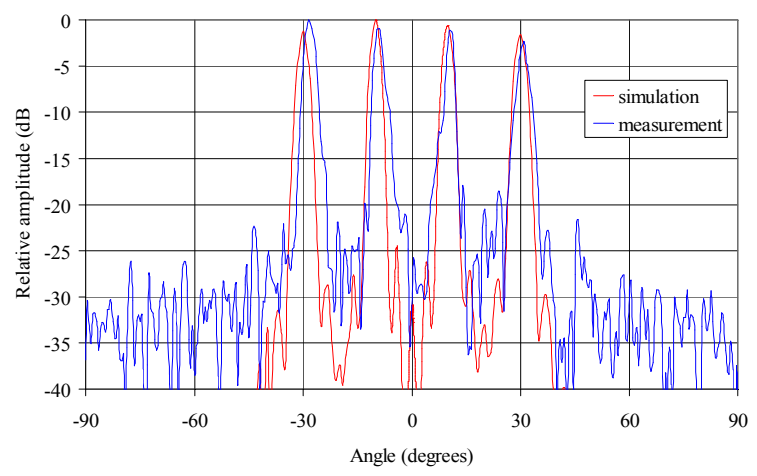

Fig. 18. Radiation pattern measurements at $94 \mathrm{GHz}$

For demonstrating the concept, we chose to place the four lobes in the same plane $\left(\varphi=45^{\circ}\right)$ with the four main radiation directions for $\theta=-30^{\circ}, 0^{\circ}, 10^{\circ}$ and $30^{\circ}$. The good agreement between measurements and simulations that can be observed in figure 18 shows that our reflect array model remains valid for more sophisticated structures.

However, depending on the positions of the beams, it is possible that no patches are present in the center of the structure. It is due to the fact that the phase range covered with rectangular patches at $94 \mathrm{GHz}$ does not exceed $320^{\circ}$ if we aim to fabricate the reflector with classical printed circuits techniques. The consequence is a $2 \mathrm{~dB}$ difference in the amplitudes of the lobes created by the surface totally filled with patches and the one with some lack of patches. More details can be found in [20].

\section{Conclusion}

This paper has investigated the modeling and performances of single layer reflectarrays in the W-band. First of all, it has been shown that a tool based on the equivalent aperture method is efficient for large reflect array modeling and design. It has been validated over a various set of structures including offset fed and multi-lobe reflectarrays. Its main advantage is its simplicity of implementation that makes it possible to simulate large structures within a short time. In particular, comparisons with measurements have been shown that the assumptions made to simplify the model were not critical for the main and first side lobes accuracy.

Thanks to this program, low-cost solutions were investigated. The influence of cell size and the primary feed were studied. It was pointed out that going below a cell size of $(\lambda / 2)$ is critical due to the fabrication tolerances, at least with classical printed circuits techniques. An original ultra-low side lobe reflectarray was designed thanks to a PSF like radiation pattern primary. In order to take advantage of the PSF, it has been shown that the reflectarray has to be fed in offset. Although the first design provided satisfying $48 \%$ aperture efficiency at $94 \mathrm{GHz}$ and $5 \% 3 \mathrm{~dB}$ gain bandwidth, results can be improved up to $61 \%$ aperture efficiency and $18 \%$ bandwidth provided that the main beam steers in the specular direction. Finally, a multi-lobe antenna was proposed for future radar application purpose.

\section{References}

[1] D. M. Pozar, S. D. Targonski, and H. D. Syrigos, "Design of millimeter wave microstrip reflectarrays", IEEE Trans. Antennas Propag., vol. 45, pp. 287-296, February 1997.

[2] W. Menzel, D. Pilz, R. Leberer, " $A 77 \mathrm{GHz} F M / C W$ radar front-end with a low profile lowloss printed antenna", IEEE Trans. Microwave Theory and Techniques, vol.47, pp.2237-2241, December 1999.

[3] S. Dieter, C. Fisher, W. Menzel, "Design of a Folded Reflectarray Antenna Using Particle Swarm Optimization", 40th European Microwave Conference, September 28 - 30, 2010, Paris, France

[4] N. Yonemoto, N. Yamamoto, K. Yamada, H. Yasui, N. Tanaka, C. Migliaccio, J-Y. Dauvignac, Ch. Pichot, "Performance of obstacle detection and collision warning system for civil helicopters", Defense and Security Symposium 2006, SPIE, AeroSense, 17-21-April 2006, Orlando, Florida, USA

[5] W. Menzel, D. Pilz, M. Al-Tikriti, "Millimeter wave folded reflector antennas with high gain, low loss and low profile", IEEE Antenna's and Propagation Magazine,vol.44, pp.24-29, june 2002

[6] J.A. Encinar, "Design of two layer printed reflectarrays using patches of variable size", IEEE Trans. Antennas Propagat., vol.49, pp.1403-1410, october 2001

[7] N. Misran, R. Cahill, and V. F. Fusco, "Design optimisation of ring elements for broadband reflectarray antenna," Proc. Inst. Elect. Eng.- Microw. Antennas Propag., vol. 150, no. 6, pp. 440-444, Dec. 2003.

[8] A. Yu, F. Yang, A.Z. Elsherbeni, J. Huang, "A single layer broadband circularly polarized reflectarray based on the element rotation technique", Antennas and Propagation Society International Symposium, 2009. APSURSI '09. IEEE

[9] W. Menzel, J. Li, S. Dieter, "Folded reflectarray antenna 
based on a single layer reflector with increased phase angle range", 3rd European Conference on Antennas and Propagation,(EuCAP 2009), 23-27 March 2009

[10] L. Matekovits, V.A. Laza, F. Vipiana, P. Pirinoli, G. Vecchi, "Multiscale analysis of array and antenna farm problems", IEEE APS Symposium, 2005

[11] Scilab www.scilab.org

[12] O. Bucci, G. Mazzarella, G. Panariello, "Reconfigurable arrays by phase-only control", IEEE Trans. Antennas Propagat, Vol. 39, pp. 919-925, July 1991.

[13] C. Han, C. Rodenbeck, J. Huang, K. Chang, "A C/Ka Dual Frequency Dual Layer Circularly Polarized Reflect array Antenna with MicrosItrip Rings Elements", IEEE Trans. Antennas Propagat, Vol. 52, No. 11, pp. 2871-2876, November 2004

[14] A. Zeitler, J. Lanteri, Ch. Pichot, C. Migliaccio, P. Feil, and W. Menzel, "Folded Reflectarrays with Shaped Beam Pattern for Foreign Object Debris Detection on Runways", IEEE Trans. Antennas Propagat. Vol.58 nº, pp. 3065-3068, 2010.R.

[15] D.Slepian and H.O Pollak, "Prolate Spheroidal wave func- tions, Fourier analysis and uncertainty I", Bell System Technical Journal, vol. 40, pp. 43-64, January 1961.

[16] H.J. Landau and H.O. Pollak. Prolate spheroidal wave functions,Fourier analysis and uncertainty, II. Bell System Technical Journal, 40:65-84, January 1961.

[17] R. Soummer et al., "Prolate apodized coronography: numerical simulations for circular apertures", Astronomy with High Contrast Imaging, C. Aime and R. Soummer (eds), EAS Publications Series (8) (2003), pp. 93-105.

[18] A. Berthon and R. P. Bills, "Integral equation analysis of radiating structures of revolution", IEEE Trans. Antennas Propagat, Vol. 37, pp. 159-170, February 1989.

[19] J.Lanteri et al., "Improvement of reflectarrays and lenses radiation pattern by prolate spheroidal functions in $\mathrm{W}$ band", EUCAP, November 2006, Nice, France.

[20] J. Lanteri, C. Migliaccio, Juha Ala-Laurinaho, Matti Vaaja, Juha Mallat, Anti V. Räisänen, "Four-Beam Reflect-Array Antenna for Mm-waves: Design and Tests in Far-Field and Near-Field Ranges", EuCAP 2009, Berlin, Germany, 23-27 March 2009. 